\title{
El Papel del MTSK como Modelo de Conocimiento del Profesor en las Interrelaciones entre los Espacios de Trabajo Matemático
}

\author{
The Role of MTSK as Model of Teachers' Knowledge in the Relationship \\ between Mathematical Working Spaces
}

\author{
Eric Flores-Medrano* \\ Miguel A. Montes ${ }^{* *}$ \\ José Carrillo $^{* * *}$ \\ Luis C. Contreras** \\ Ma Cinta Muñoz-Catalán \\ Mar Liñán
}

\begin{abstract}
Resumen
El profesor desempeña un papel crucial en la articulación de los Espacios de Trabajo Matemático. El modelo de conocimiento del profesor que aquí se presenta (denominado Mathematics Teacher's Specialised Knowledge) propone una estructuración de dicho conocimiento basada en la noción de 'especialización'. Esta estructuración,
\end{abstract}

\footnotetext{
* Licenciado en Física y Matemáticas, Instituto Politécnico Nacional (IPN), México; Maestro en Ciencias con especialidad en Matemática Educativa, Centro de Investigación y de Estudios Avanzados (Cinvestav), México, y Doctor en Didáctica de la Matemática, Universidad de Huelva (UHU), España. Dirección postal: Facultad de Ciencias de La Educación, Campus El Carmen, Avenida del 3 de Marzo, s/n, 21071. Huelva, España. E-mail: ericfm_0@ hotmail.com.

* Licenciado en Matemáticas por la Universidad de Sevilla (US), Doctor por la Universidad de Huelva (Didáctica de las Matemáticas, UHU), Profesor sustituto Interino de Didáctica de la Matemática, Universidad de Huelva (UHU), España, Dirección postal: Facultad de Ciencias de La Educación, Campus El Carmen, Avenida del 3 de Marzo, s/n, 21071. Huelva, España. E-mail: miguel.montes@ ddcc.uhu.es.

${ }^{* * * *}$ Licenciado en Matemáticas y Doctor en Filosofía y Ciencias de la Educación, Universidad de Sevilla (US), España. Catedrático de Universidad de Didáctica de la Matemática, Universidad de Huelva (UHU), Huelva, España. Dirección postal: Facultad de Ciencias de La Educación, Campus El Carmen, Avenida del 3 de Marzo, s/n, 21071. Huelva, España. E-mail: carrillo@uhu.es.

Licenciado en Matemáticas, Universidad de Sevilla (US), y Doctor en Psicopedagogía, Universidad de Huelva (UHU), España. Profesor Titular de Universidad de Didáctica de la Matemática, Universidad de Huelva (UHU), Huelva, España. Dirección postal: Facultad de Ciencias de la Educación, Campus El Carmen, Avenida del 3 de Marzo, s/n, 21071. Huelva, España. E-mail: 1carlos@uhu.es.

Licenciada en Psicopedagogía y Doctora por la Universidad de Huelva (Didáctica de las Matemáticas, UHU), España. Profesora Ayudante Doctora de Didáctica de las Matemáticas, Universidad de Sevilla (US), Sevilla, España. Dirección postal: Facultad de Ciencias de la Educación, C/ Pirotecnia, s/n, 41013 Sevilla, España. E-mail: mcmunozcatalan@us.es.

Licenciada en Matemáticas, Universidad Autónoma de Madrid (UAM) y Máster en Investigación en la Enseñanza y el Aprendizaje de las Ciencias Experimentales, Sociales y Matemáticas, Universidad de Huelva (UHU). Profesora Didáctica de las Matemáticas, Centro de Estudios Universitarios Cardenal Spínola (CEU), Profesora Didáctica de las Matemáticas Universidad de Sevilla (US), Sevilla, España. Dirección postal: Fundación San Pablo Andalucía CEU Campus Universitario CEU, Glorieta Ángel Herrera s/n, 41930- Bormujos, Sevilla, España. E-mail: mlinan@us.es.
} 
particularmente la consideración de sus componentes (subdominios), permite comprender el conocimiento que el profesor pone en juego en la transición entre los distintos Espacios (idóneo, personal y de referencia) de Trabajo Matemático, así como las relaciones entre estos.

Palabras clave: Espacios de Trabajo Matemático. Conocimiento Especializado del Profesor de Matemáticas. Relaciones entre ETM y MTSK.

\begin{abstract}
The teacher plays a crucial role in articulating Mathematical Working Spaces. The model of teacher knowledge, which is presented here (referred Mathematics Teacher's Specialized Knowledge), proposes a structuring of such knowledge based on the concept of 'specialization'. This organization, particularly considering its components (sub-domains), provides insight into the knowledge that the teacher brings into play in the transition between different (appropriate, personal, and reference) Mathematical Working Spaces, and the relationships between them.
\end{abstract}

Keywords: Mathematical Working Spaces. Mathematics Teacher's Specialized Knowledge. Relationships between MWS and MTSK.

\title{
1 Introducción
}

La noción de Espacio de Trabajo Matemático [ETM] (KUZNIAK, 2011) constituye un modelo explicativo del trabajo matemático del alumno en un marco escolar, y emerge como ampliación de los estudios sobre los Espacios de Trabajo Geométricos. Como señalan Kuzniak y Richard (2014), la investigación didáctica, en el marco de una enseñanza que favorezca el desarrollo del trabajo matemático del alumno, debe interrogarse sobre este trabajo desde el punto de vista de la organización de la enseñanza por parte del profesor; por ello, en la configuración de los elementos que configuran los ETM, aparece la figura del profesor como agente responsable de su generación. En el cuarto simposio Espacio de Trabajo Matemático, celebrado en San Lorenzo del Escorial (Madrid), se abordó la potencialidad de este modelo como herramienta de análisis del quehacer del estudiante en el aula de matemáticas, el papel de las herramientas tecnológicas en la transformación de espacios de trabajo de los estudiantes, y en el propio trabajo matemático, así como en aspectos sociales e institucionales en la constitución de los ETM. Dentro esta última temática, la formación del profesor se señalaba como pilar institucional fundamental y, por ello, se consideró necesario profundizar en la figura del profesor en el marco del ETM. Por ello, una de las temáticas de reflexión del cuarto simposio Espacio de Trabajo Matemático fue la génesis y desarrollo del trabajo matemático: el papel del profesor y las interacciones (GÓMEZ-CHACÓN; ROMERO; CARRILLO, 2015). 
Nuestra aportación a esta tarea ha sido intentar comprender el trabajo matemático desde la perspectiva del profesor. En concreto, interesa el papel del profesor, y en particular de su conocimiento, en la configuración del ETM idóneo, en la evolución de éste ajustándose a las restricciones locales y en cómo el profesor interpreta el ETM de referencia.

La especificidad del conocimiento del profesor en relación con la enseñanza de la matemática, frente a otras disciplinas, ha sido objeto de estudio por la comunidad científica (PONTE; CHAPMAN, 2006). Algunos modelos, como el denominado Mathematical Knowledge for Teaching, han señalado la existencia de un tipo de conocimiento puramente matemático que es diferente para el profesor respecto a otros profesionales (BALL; THAMES; PHELPS, 2008). Desde la perspectiva de que esta especialización abarca a todo el conocimiento del profesor de matemáticas, Carrillo, Climent, Contreras y Muñoz-Catalán (2013) plantean un modelo en el que, con fines analíticos, se caracterizan conocimientos de diferente naturaleza. En este trabajo elegimos este último modelo, denominado Mathematics Teachers' Specialised Knowledge por su consideración del contenido matemático en sus diferentes subdominios. Describiremos con detalle cada uno de los elementos de este modelo y estableceremos cuál es la relación del modelo en su conjunto con las nociones de Espacio de Trabajo Matemático personal, idóneo y de referencia.

\section{El marco de los Espacios de Trabajo Matemático}

El modelo de Espacio de Trabajo Matemático (ETM) es una generalización de los Espacios de Trabajo Geométrico descritos por Montoya-Delgadillo, Mena-Lorca y MenaLorca (2014). Su objetivo en la investigación es mejorar la comprensión de los fenómenos didácticos en torno al trabajo de los individuos con actividades matemáticas en el contexto escolar (KUZNIAK; RICHARD, 2014).

Bajo la noción de ETM se esconde una pluralidad. Si enfocamos la mirada en el punto más lejano (metafóricamente) de la actividad concreta del aula, nos encontramos con el ETM de referencia, en el cual se hace presente la idea de paradigma. Un paradigma se instituye cuando una comunidad de individuos acuerda formular problemas y organizar sus soluciones conforme a una determinada forma de entender las cosas (KUZNIAK; RICHARD, 2014). El espacio de trabajo paradigmático que define esa comunidad se denomina ETM de referencia, del cual emergerá el ETM de referencia de cada profesor como proyección o interpretación del anterior (nos referimos a la interpretación del ETM de referencia que cada profesor realiza). 
Cuando pensamos en la actividad matemática en una escuela, representada genuinamente por la resolución de un problema, se necesita elaborar un ETM idóneo que permita la implicación del alumno en dicha resolución. Para ello, este ETM idóneo debe permitir trabajar en el paradigma correspondiente a la problemática concreta y estar construido de modo que sus diferentes componentes estén organizadas de forma válida (KUZNIAK; RICHARD, 2014). En clase, la concepción del ETM idóneo depende del ETM personal del profesor; y, puesto que el problema se le plantea a un estudiante, su tratamiento matemático por parte del mismo va a estar condicionado por el ETM personal del alumno; así, el ETM idóneo se ve alterado por condicionantes locales (KUZNIAK; RICHARD, 2014, p. 10):

De esta manera, el trabajo matemático en un marco escolar se puede describir gracias a tres niveles de ETM: la matemática considerada por la institución, que se describe en el ETM de referencia, desarrollado por el profesor hasta alcanzar un ETM idóneo que permita un establecimiento efectivo en clase, donde cada alumno trabaja para su ETM personal.

La elección y la organización de las tareas propuestas a los alumnos por los profesores son esenciales en la constitución del ETM idóneo. [...] La observación de la actividad de los alumnos permitirá identificar sus ETM personales, identificando posibles subconjuntos de prácticas estables.

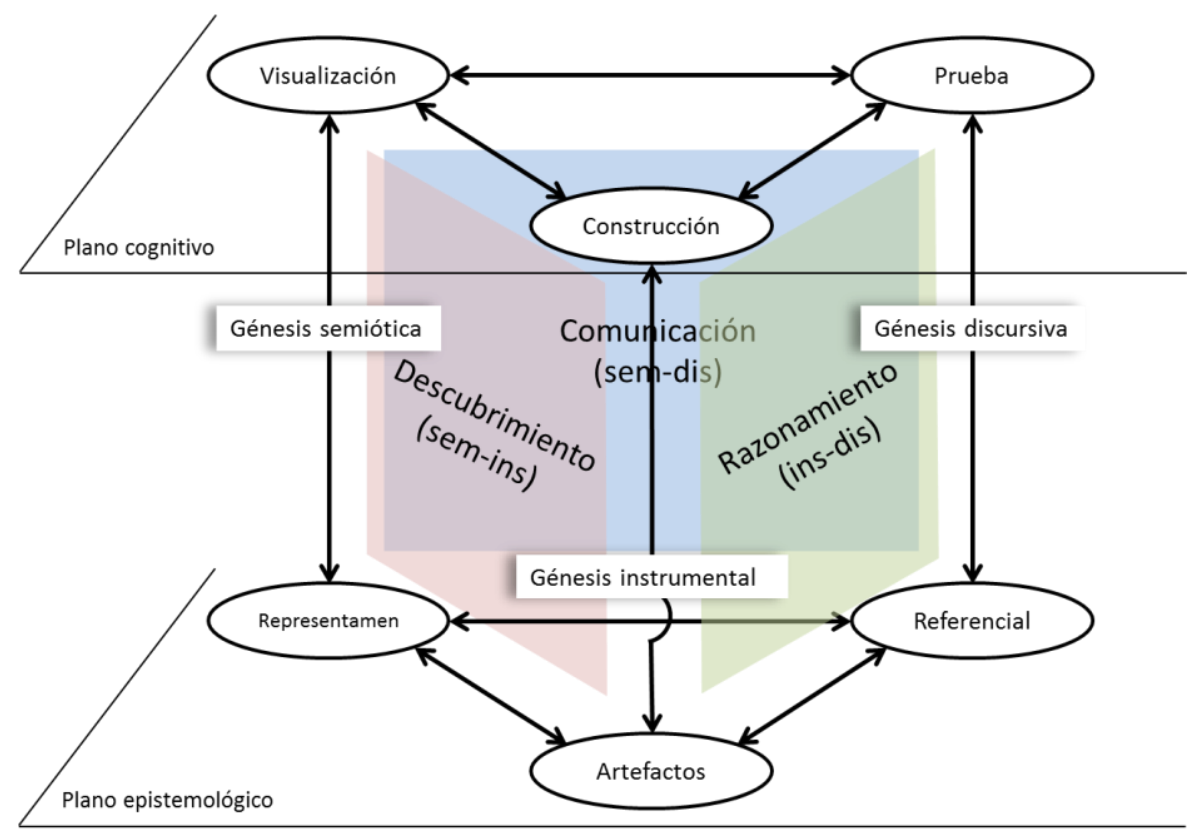

Figura 1- Los planos, polos y génesis del Espacio de Trabajo Matemático (KUZNIAK; RICHARD, 2014)

Los Espacios de Trabajo Matemático se componen de dos planos, el epistemológico y el cognitivo. El modelo integra estos dos planos mediante la consideración de relaciones entre elementos del trabajo matemático en cada uno de estos. A dichos elementos se les llama polos y las relaciones son nombradas génesis (RAUSCHER; ADJIAGE, 2014). En la Figura 1 se muestra el esquema de los Espacios de Trabajo Matemático. En este se incluyen tres planos 
verticales, los cuales explican, de una manera más general, las relaciones que existen entre los polos y génesis del ETM.

El plano de descubrimiento se apoya en la génesis semiótica e instrumental para identificar y explorar objetos en la solución de los problemas matemáticos (KUZNIAK; RICHARD, 2014). La génesis instrumental se refiere a los mecanismos por medio de los cuales un artefacto se convierte en un instrumento y así puede integrarse en una persona con la finalidad de construir conocimiento matemático (MONTOYA-DELGADILLO; MENALORCA; MENA-LORCA, 2014).

El plano de comunicación se refiere al tratamiento, interacción y comunicación del contenido matemático que está siendo trabajado por una persona. Interrelaciona el referencial (conjunto de conocimientos teóricos) con la prueba a través de la génesis discursiva de la misma prueba, en la que el individuo "utiliza las propiedades en el referencial teórico para ponerlas al servicio del razonamiento matemático y de una validación" (KUZNIAK; RICHARD, 2014, p. 11). Asimismo, para que el proceso de comunicación tenga consistencia, se considera que la persona establece una génesis semiótica, que "asegura el establecimiento de la relación entre sintaxis, semántica, función y estructura de los signos vehiculados" (KUZNIAK; RICHARD, 2014, p.11).

Con respecto al plano del razonamiento, Kuzniak y Richard (2014, p. 12) señalan que "está fundado en la justificación de los descubrimientos, que articula las génesis instrumental y discursiva".

\section{MTSK: un modelo de conocimiento especializado del profesor de matemáticas}

Coincidimos con otros autores, cuyo pionero es Shulman $(1986,1987)$, en que para la enseñanza de una materia (en este caso matemáticas) el profesor necesita un conocimiento específico, y asociamos esa especificidad a la enseñanza de la misma.

En las investigaciones que hemos desarrollado colectivamente en el grupo SIDM $^{1}$ sobre el conocimiento profesional del profesor de matemáticas, el trabajo con los modelos existentes nos ha permitido conocer sus limitaciones y potencialidades y, como consecuencia, nos ha llevado a proponer un modelo centrado en lo que es específico del profesor de matemáticas, dejando de lado aspectos del conocimiento que son compartidos con profesores

\footnotetext{
${ }^{1}$ SIDM es el Seminario de Investigación en Didáctica de la Matemática. Este grupo tiene su sede en la Universidad de Huelva (España). En él participan investigadores de universidades de España, Portugal, México, Chile, Perú, Ecuador y Brasil, entre los cuales se encuentran los autores de este trabajo. 
de otras disciplinas. Proponemos, así el modelo Mathematics Teacher's Specialised Knowledge (MTSK), en el cual consideramos, siguiendo la línea de Shulman (1986), dos grandes dominios de conocimiento: el conocimiento de las matemáticas y el conocimiento de aspectos relacionados con el contenido matemático como objeto de enseñanza-aprendizaje (conocimiento didáctico del contenido). Entendemos que la especificidad del conocimiento del profesor no se inscribe únicamente en el dominio matemático, sino que permea también el conocimiento didáctico del contenido. Incluimos asimismo las creencias, en las que no nos extenderemos en este artículo. El modelo queda reflejado en la figura 2, en la que las siglas se corresponden con los nombres de dominios y subdominios en inglés (MK: Mathematical Knowledge; KoT: Knowledge of Topics; KSM: Knowledge of the Mathematical Structure; KPM: Knowledge of Practices in Mathematics; PCK: Pedagogical Content Knowledge; KMT: Knowledge of Mathematics Teaching; KFLM: Knowledge of Features of Learning Mathematics; KMLS: Knowledge of Mathematics Learning Standards; ver CARRILLO et al., 2013).

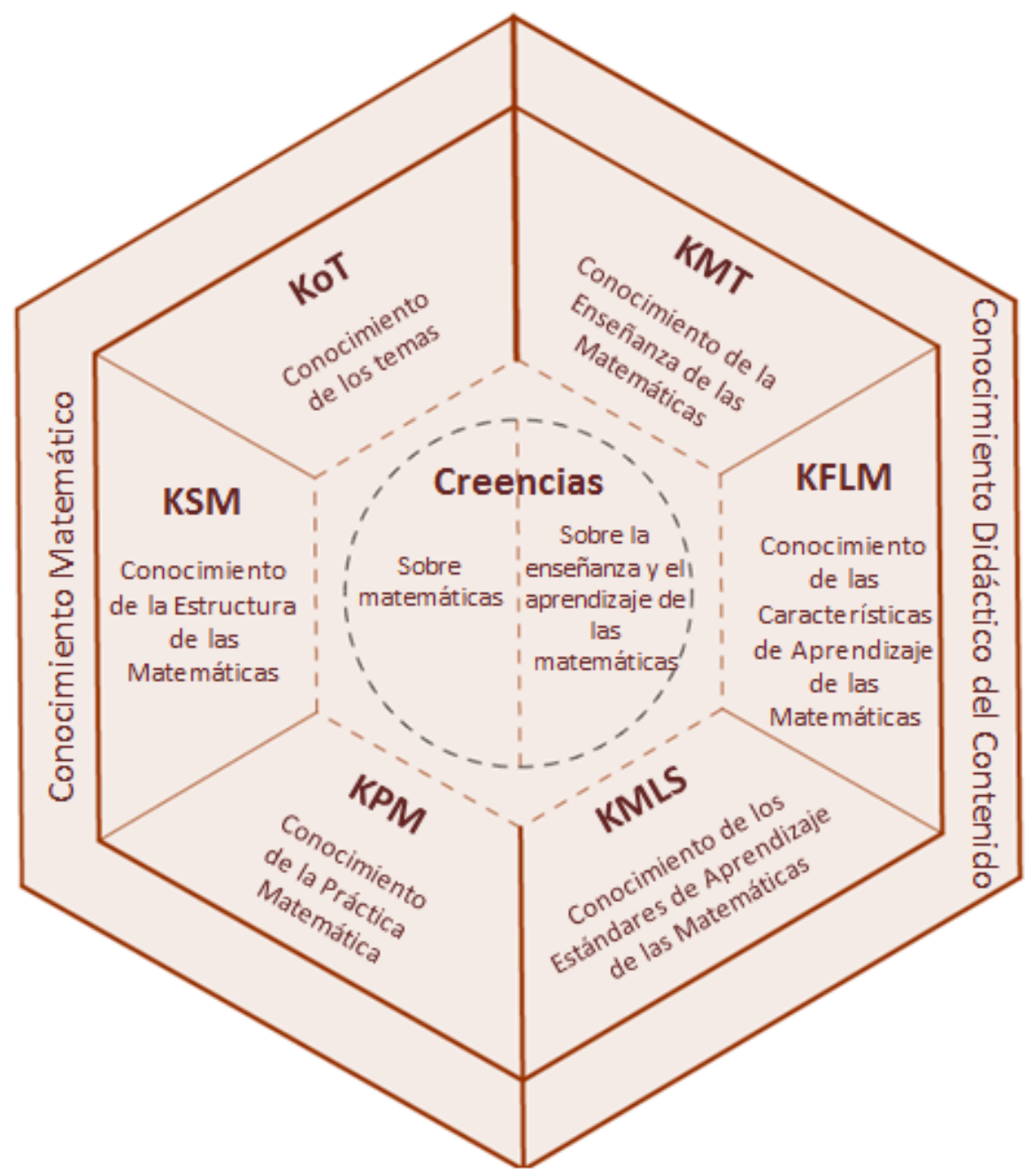

Figura 2- Subdominios del MTSK (CARRILLO et al., 2013) 


\subsection{Conocimiento de las Matemáticas (MK)}

Proponemos tres subdominios que componen y dan sentido al conocimiento matemático del profesor de matemáticas: el conocimiento profundo del contenido matemático en sí (el conocimiento de los temas matemáticos), de su estructura (conocimiento de la estructura matemática) y de cómo se procede y produce en matemáticas (conocimiento de la práctica matemática).

\subsubsection{Conocimiento de los temas matemáticos (KoT)}

El conocimiento de los temas no se refiere solamente al conocimiento de la matemática como disciplina, sino que incluye a la matemática escolar. Así, describe qué y cómo conoce el profesor de matemáticas los temas que va a enseñar; supone conocer de manera fundamentada los contenidos matemáticos (conceptos, procedimientos, hechos, reglas, teoremas, etc.) y sus significados. Integra el contenido que queremos que aprenda el alumno, con un nivel de profundización sustancialmente mayor.

El término temas se refiere a los contenidos provenientes de los bloques de conocimiento tradicionalmente considerados en matemáticas. Como referente, tomamos las áreas propuestas por el NCTM (2000) en los estándares matemáticos: números y operaciones, álgebra, geometría, medida, análisis de datos y probabilidad, los cuales están relacionados entre sí. Los temas son los componentes de estas grandes ramas y pueden variar de acuerdo al currículo de cada país.

En el conocimiento del profesor sobre los temas consideramos el conocimiento de los significados asociados al contenido, de los fenómenos que le dan sentido (algunos ligados a su origen), de las aplicaciones del contenido (en la matemática o en otras áreas), de las definiciones e imágenes de un concepto, de las propiedades y su fundamentación, de las representaciones del contenido y de los procedimientos.

Por ejemplo, incluimos en el KoT el conocimiento del profesor de los atributos relevantes, irrelevantes e incorrectos de una figura geométrica determinada; saber la definición de una determinada figura en función de la clasificación inclusiva o excluyente que la origina; identificar objetos de la vida real que una determinada figura modeliza y/o explica.

En este subdominio integramos las relaciones intra-conceptuales (FERNÁNDEZ; FIGUERAS; DEUFELOU; MARTÍNEZ, 2011), al considerarlas como un conocimiento profundo del tema, más que relaciones con otros contenidos (relaciones inter-conceptuales, 
que, como veremos a continuación, estarán incluidas en el Conocimiento de la Estructura Matemática).

Nótese que se trata de un conocimiento fundamentado, que supone conocer el contenido desde un punto de vista más profundo del que corresponde al nivel de aprendizaje en cuestión. Además, se define de modo intrínseco (refiriéndonos exclusivamente al conocimiento matemático del profesor de matemáticas, y no en función de si ese conocimiento es compartido o no con otros profesionales, como se hace en el MKT en relación con la caracterización del conocimiento común y el especializado - BALL et al., 2008).

\subsubsection{Conocimiento de la estructura matemática (KSM)}

El conocimiento de la estructura pretende recoger el conocimiento del profesor sobre la red de relaciones de contenidos matemáticos, lo que supone de hecho su conocimiento de la estructura del edificio matemático. Es el conocimiento sobre las relaciones entre distintos contenidos (MONTES; AGUILAR; CARRILlO; MUÑOZ-CATALÁN, 2013), ya sea del curso que está impartiendo o con contenidos de otros cursos o niveles educativos. Se trata específicamente de conexiones entre temas matemáticos. Se contemplan dos situaciones, no excluyentes entre sí, que generan conexiones: la temporalidad, como visión secuenciadora (no curricular) que genera conexiones de complejización y simplificación; y la delimitación de objetos matemáticos, que genera conexiones inter-conceptuales (MARTÍNEZ; GINÉ; FERNÁNDEZ; FIGUERAS; DEULOFEU, 2011).

Incluimos en este subdominio el conocimiento de ideas principales o transversales a distintos contenidos (como el conocimiento lógico matemático de la clasificación que está en el sustrato de todos los tópicos matemáticos), de relaciones entre distintos temas (por ejemplo, entre fracciones, decimales y porcentajes) y de relaciones dadas por simplificación o complejización del tema (por ejemplo, entre las series de tipo recursivo, la secuencia numérica y las progresiones aritméticas). La consideración de la matemática avanzada desde un punto de vista elemental (simplificación) y viceversa (complejización) supone una aportación relevante del modelo en lo que respecta al estudio de cómo el profesor conecta temas matemáticos.

\subsubsection{Conocimiento de la práctica matemática (KPM)}


Además de conocer los núcleos de contenidos matemáticos y sus relaciones, el profesor debe poseer conocimiento de cómo se genera conocimiento matemático, cuáles son las reglas de sintaxis de la disciplina. Este subdominio incluye, por ejemplo, conocimiento de cómo se define en matemáticas, la diferencia entre una demostración, una prueba y una comprobación, el valor en ésta de los ejemplos y en el planteamiento de una conjetura, de distintos tipos de demostraciones y su campo de acción.

Por ejemplo, saber que la búsqueda de un ejemplo, puede suponer en unos casos una mera comprobación de una propiedad y, en otros casos, una demostración. Así, en el primer caso, tendríamos que el ejemplo de $5+2=2+5$ permite comprobar la propiedad conmutativa de la suma, pero no demuestra su validez en cualquiera de los conjuntos numéricos. Sin embargo, el ejemplo de $5-2 \neq 2-5$ es suficiente para demostrar que dicha propiedad no es válida para la operación de la resta, además de que la resta no es una operación interna en el conjunto de los números naturales.

Hemos de aclarar que la práctica a la que se refiere este subdominio es la práctica matemática, no la práctica de la enseñanza de la matemática; y las formas de proceder se refieren a las formas de proceder en matemáticas (conocimiento de heurísticos para resolver problemas, conocimiento de las situaciones que requieren de un uso de pensamiento inductivo o de pensamiento deductivo), y no a saber utilizar los procedimientos con objetos matemáticos (lo cual está contemplado en el conocimiento del tema).

\subsection{Conocimiento Didáctico del Contenido (PCK)}

Consideramos tres subdominios con los que reconocemos el conocimiento que tiene el profesor acerca del contenido como objeto de aprendizaje (conocimiento de las características de aprendizaje de las matemáticas), como objeto de enseñanza (conocimiento de la enseñanza de las matemáticas) y desde el punto de vista de lo que se debe/puede alcanzar en un determinado momento escolar (conocimiento de los estándares de aprendizaje de las matemáticas).

\subsubsection{Conocimiento de las características de aprendizaje de las matemáticas (KFLM)}

Tanto en este subdominio como en el que sigue hemos interpretado el carácter especializado del conocimiento del profesor de matemáticas en el sentido de que el 
conocimiento que nos interesa no es la intersección de conocimiento del contenido y de un conocimiento pedagógico general (referido en este caso a aprendizaje y, en el que sigue, a enseñanza), sino el conocimiento de cómo aprenden los estudiantes los contenidos matemáticos (o aspectos de la enseñanza intrínsecamente ligados a los contenidos matemáticos).

En este subdominio incorporamos conocer cuáles son los modos habituales de razonamiento de los alumnos en determinados contenidos, cuáles son sus dificultades, los aspectos que les resultan más comprensibles, así como cuáles les suelen resultar más y menos atractivos. Este conocimiento puede estar fundamentado en teorías personales del profesor o institucionalizadas. El profesor puede conocer teorías de aprendizaje - como el modelo de Sfard (1991) de comprensión de un objeto matemático - que sustenten su comprensión del aprendizaje de determinados contenidos matemáticos. Igualmente, damos cabida a que la fundamentación de su conocimiento tome la forma de teorías prácticas personales (AZCÁRATE, 1999) que provienen en gran parte de su experiencia docente.

Por ejemplo, el conocimiento que tiene el profesor acerca de cuáles son las fases a través de las cuales los niños construyen su comprensión del Sistema de Numeración Decimal (LLINARES, 2001); de cuáles son los errores habituales que se cometen en cada una de ellas y del tipo de algoritmo formal o informal con el que pueden acometer las operaciones en cada fase.

\subsubsection{Conocimiento de la enseñanza de las matemáticas (KMT)}

Consideramos en este subdominio el conocimiento que tiene el profesor sobre modos de presentar el contenido y su potencial para la instrucción (incluyendo el conocimiento de ejemplos adecuados para cada contenido, intención o contexto determinado), así como el conocimiento de la potencialidad de los recursos y materiales didácticos respecto de la actividad matemática.

Los ejemplos y representaciones del contenido son considerados desde el punto de vista de su potencial para el aprendizaje (a diferencia de las representaciones consideradas en el conocimiento del tema, desde el punto de vista de su potencial matemático). Por ejemplo, para trabajar las propiedades del Sistema de Numeración Decimal, incluimos aquí conocer la potencialidad de los bloques de base diez para la comprensión del principio de agrupamiento de dicho sistema y la utilidad de los ábacos para trabajar el valor de posición. 
Al igual que en el subdominio anterior, puede ser un conocimiento fundamentado en teorías fruto de la investigación en Educación Matemática o en la observación y reflexión de la actividad matemática en el aula.

\subsubsection{Conocimiento de los estándares de aprendizaje de las matemáticas (KMLS)}

Recoge el conocimiento del profesor sobre lo que está estipulado que aprenda un estudiante y el nivel de profundidad y manejo con el que se espera que lo aprenda en un determinado momento escolar, así como secuenciaciones del contenido y las razones que lo sustentan. Este conocimiento se puede fundamentar, además de en los documentos curriculares correspondientes, en otros documentos sobre estándares de aprendizaje e investigaciones que aportan recomendaciones al respecto.

\subsection{El papel de las creencias del profesor en el MTSK (Beliefs)}

Somos conscientes de que la práctica del profesor tiene detrás una filosofía de las matemáticas que la respalda (THOM, 1973). Entendemos que esta filosofía contiene un conjunto de concepciones y creencias del profesor (THOMSON, 1992) acerca de las matemáticas, su enseñanza y su aprendizaje. Dichas concepciones permean a su conocimiento en cada uno de los subdominios.

Buscamos construir imágenes cada vez más precisas que permitan interpretar la práctica del profesor a la luz de aspectos que influyen en ella basándonos en los conocimientos que sustentan dicha práctica. En ese sentido, interpretamos las concepciones del profesor en el sentido de que éstas pueden conformar un sistema que sea explicado en sí mismo (Sensible System Framework, LEATHAM, 2006). Somos conscientes de que las concepciones representan una predisposición a través de las acciones y que no pueden ser directamente observadas o medidas, solamente inferidas. Al igual que el resto de elementos en el modelo, las concepciones son consideradas con fines analíticos.

\section{Cómo influye el MTSK en las interrelaciones entre los distintos espacios de trabajo matemático}

Iniciaremos este apartado con un ejemplo, cuya discusión evidenciará cómo el conocimiento del profesor se usa para gestionar los diferentes ETM. Una profesora de tercer 
curso de Educación Primaria genera, por primera vez en su curso, un espacio de trabajo matemático sobre los conceptos de recta, semirrecta o segmento (LIÑÁN; MONTES; CONTRERAS, 2015). Dicha generación parte del ETM de referencia de la profesora, teniendo en cuenta diversos elementos, entre otros el currículo y el libro de texto. Estos no contemplan la idea de infinito (actual o potencial); es decir, en los referentes que contempla el profesor, no se tiene en cuenta este contenido y éste, por tanto, no considera esa idea de infinito en el ETM idóneo para la sesión, lo que supone una decisión mediada por la organización y profundidad de su conocimiento especializado como profesor de matemáticas. En la configuración del ETM idóneo ha intervenido el conocimiento (MTSK) de la maestra. De modo particular, el KMT y el KFLM han actuado como adaptadores del ETM de referencia al ETM idóneo. En el KFLM, por ejemplo, podemos ubicar el conocimiento del profesor sobre cómo producen los alumnos las génesis semiótica, instrumental y discursiva. Y en relación con este conocimiento situamos el conocimiento de estrategias y recursos que favorecen dichas génesis (KMT). Asimismo, si nos fijamos en la génesis discursiva, apreciamos la influencia del conocimiento del profesor sobre el modo de proceder en matemáticas (KPM). En la transcripción siguiente, notamos M por Maestra y A por Alumno/a, acompañado de un número que indica el orden de los diferentes alumnos que intervienen:

M: $\quad$ (dibuja un segmento rectilineo paralelo a la horizontal con inicio y fin, marcando los puntos) Esto no es (borra los puntos marcados). No tiene principio ni fin (amplía el segmento hasta los extremos de la pizarra y hace un gesto con las manos indicando que continuaría por ambos lados de la pizarra). No sabemos ni dónde empieza ni dónde acaba, no tiene curvas ni ángulos. Es como cuando vamos por la carretera y vemos los cables de la luz, no los que están así (hace un gesto curvo con la mano) sino los que están más tensos. Los vemos y es una recta que no sabemos dónde empieza ni dónde acaba.

A1: La carretera también.

M: $\quad$ Bueno las carreteras tienen curvas. Si tienen curvas ya no es una recta. Bueno, pues vamos a poner en el cuaderno: recta, y ponemos las características: es una línea de puntos que no tiene ni principio ni fin y que no tiene líneas curvas ni ángulos.

Ahora vamos a ver lo que es una semirrecta. Tenemos una recta y la cortamos. Como una recta es infinita, la cortamos y tenemos dos semirrectas. Según esto, A2, ¿qué será una semirrecta?

A2: $\quad$ Un punto que divide a la recta en dos partes iguales.

M: $\quad$ Entonces, si tengo otra recta y le hacemos un punto aquí (dibuja una recta en la pizarra y un punto, que borra y luego vuelve a pintar en otro lugar), como es infinita, pues una semirrecta estaría aquí (señala) y la otra desde el punto hasta el infinito, las dos semirrectas. Definiríamos un punto y las dos semirrectas, que son infinitas.

Nota: El libro establece, en la definición de semirrecta: "un punto divide a una recta en dos partes iguales, llamadas semirrectas, con principio pero si fin", y la maestra la lee. Obvia, al volver a dibujarla en la pizarra, la afirmación "dos partes iguales".

M. $\quad$ Pues así, aquí empieza esta semirrecta y aquí la otra.

A3. Pero una es más larga que la otra, ¿no?

M. Bueno, es que son infinitas, y el infinito...Por eso he puesto yo distintos puntos, me da igual dónde esté el punto, son infinitas... 
Respecto a su conocimiento, observamos que el profesor posee diferentes grados de conciencia de la naturaleza del infinito como sustento epistemológico de algunas características de la recta (KoT, KSM), de la práctica de generalización que se induce al representar de forma finita conjuntos no acotados (KPM), o de las dificultades de los alumnos al lidiar con conceptos en los que el infinito está presente (KFLM).

La manera en la que se desarrolla el trabajo matemático in situ, unida a su conocimiento especializado, lleva a la maestra a reconsiderar sus decisiones acerca de la elección del ETM idóneo, cuando la representación que usa para la recta entra en conflicto con las limitaciones de la pizarra, o cuando, a la hora de definir semirrecta, comprueba las dificultades que implican marcar en la mitad (en vez de otro punto de la misma) de la recta representada, las dos semirrectas que de ella devienen, con el debate que supone la comparación entre las mismas. Los distintos significados de infinito que emergen o que el profesor detecta que pueden emerger de manera natural, así como los efectos que la tarea matemática produce en el trabajo matemático de los alumnos, implicará una potencial reorganización de algunos aspectos de los futuros ETM que el profesor gestionará, especialmente aquellos en los que detecte una posible emergencia de diferentes reflexiones sobre el infinito, tanto suyas propias, como de sus estudiantes. De este modo, el ETM idóneo se va modificando con la ayuda del MTSK de la maestra y a partir de los ETM de los estudiantes. La influencia de los ETM de los estudiantes no es homogénea ni lineal. La maestra, a veces, adapta su ETM idóneo a raíz del ETM de un solo estudiante, mientras que en otras ocasiones es una especie de media de los ETM de los estudiantes de la clase o de un grupo de ella lo que toma en cuenta para realizar su adaptación. Asimismo, el MTSK del profesor se verá modificado, se ampliarán y profundizarán algunos aspectos de su conocimiento, lo que se puede ver reflejado en los referentes que considere y en su propia forma de trabajar determinadas actividades matemáticas.

Para reflejar esta perspectiva de forma esquemática, proponemos un esquema (Figura 3) donde se reflejan las relaciones entre MTSK y los diferentes ETM. Con cada uno de los prismas triangulares que aparecen en la Figura 3 hemos representado de manera simplificada el modelo de ETM (Fig. 1) para centrar la atención en los planos epistemológico y cognitivo como parte de un modelo analítico de los procesos integrados de trabajo matemático. Dichos planos están representados como bases de los prismas y las caras laterales conforman los planos de razonamiento, comunicación y descubrimiento. 


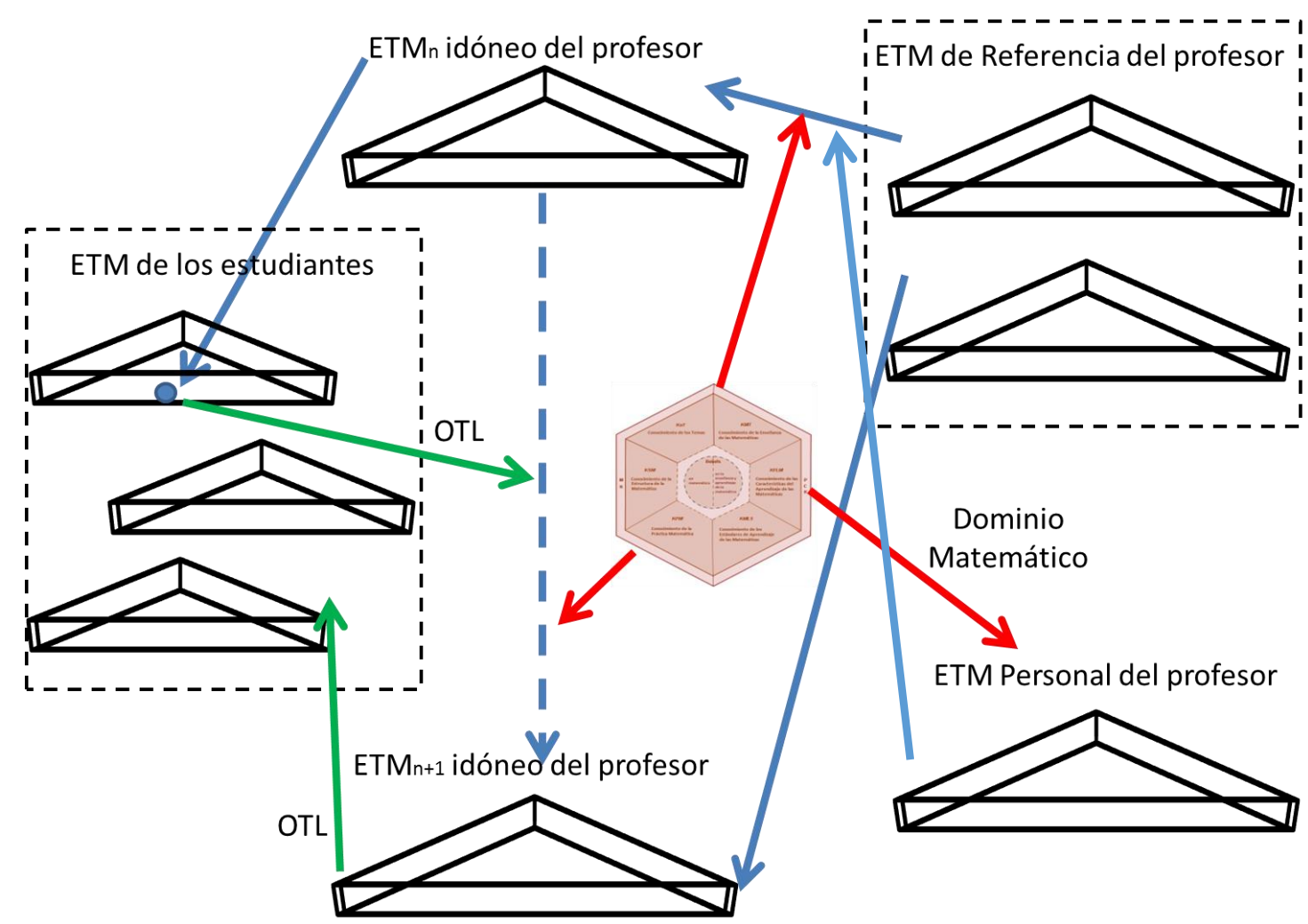

Figura 3- Esquema de relaciones entre el MTSK y los Espacios de Trabajo Matemático.

La parte derecha de este esquema pone de relieve las dos fuentes del ETM de referencia; por un lado el referente puramente matemático, como saber sabio, y, por otro, su concreción en el profesor, como el conocimiento matemático que éste tiene y que supone, de hecho, una interpretación del primero, y en el que influye el ETM de referencia institucional. Las flechas que parten de la representación del MTSK en el esquema indican la relación del conocimiento especializado del profesor de matemáticas, en su conjunto, con los diferentes ETM. Debido a que los referentes que el profesor puede considerar para conformar, gestionar y trabajar con actividades matemáticas tienen una influencia totalmente ligada al conocimiento matemático, es posible establecer relaciones entre dichos referentes y los elementos de los subdominios que forman parte del conocimiento matemático considerado en el MTSK. El conocimiento de los temas, de la estructura matemática y de la práctica matemática (subdominios KoT, KSM y KPM) están estrechamente relacionados con el ETM de referencia y con las concreciones o adaptaciones de este en el ETM personal y en el ETM idóneo. Entiéndase que, al mencionar la estructura o la práctica matemática, no estamos adoptando una estructura o una práctica particulares, es decir, no estamos proponiendo un referente epistemológico único o una concepción determinada de la matemática. Pensamos que este modelo, el MTSK, puede utilizarse desde distintas perspectivas epistemológicas, aunque nosotros compartimos una concepción dinámica de la matemática (ERNEST, 1991). 
El ETM de referencia, de naturaleza dinámica, se representa en el esquema con varios prismas, que reconocen las distintas fuentes que sirven de referente para el profesor, así como su capacidad de verse modificados en el tiempo por diferentes factores.

En un profesor determinado, y en un momento específico de su actividad docente, su conocimiento profesional, y en particular su conocimiento especializado como profesor de matemáticas, media para transformar este ETM de referencia (del profesor) en el ETM idóneo de ese momento. Esta transformación puede analizarse en función de las decisiones que el profesor adopta sobre el contenido matemático que se gestionará en el espacio de trabajo correspondiente, y que vienen mediadas por diversos subdominios de su conocimiento especializado (nivel de profundidad con el que se pretende tratar el contenido, relaciones que se pondrán en juego, elementos sintácticos que decide tratar, recursos que son adecuados para el momento,...), subdominios que podrán potencialmente, a su vez, retroalimentarse de los resultados de la reflexión sobre el trabajo matemático que se desarrolle en el aula.

El ETM idóneo, determinado por el profesor y tomado en consideración en cada momento, también es dinámico (lo que representamos con los respectivos subíndices), y depende en gran medida (aunque no solo) del conocimiento especializado que ponga en juego el profesor, del trabajo matemático que caracteriza la actividad del aula, de los ETM de los estudiantes, de la detección y aprovechamiento de las oportunidades de aprendizaje (representadas en el esquema como oportunidades de aprendizaje-OTL), así como del ETM de referencia y personal del profesor. De esta manera queremos destacar los factores que pueden modificar el ETM idóneo, que puede relacionarse con la forma de trabajo prevista para implementarse en el aula, según se va desarrollando el propio trabajo matemático.

\section{Consideraciones finales}

Parece evidente el papel del profesor en el diseño y gestión del ETM idóneo que propicie el trabajo matemático de los alumnos. El conocimiento del profesor juega a su vez un papel central en dichas tareas, de modo que modelos analíticos que pongan el foco en la especificidad de la matemática nos ayudarán a comprender cómo se configura dicho ETM idóneo. En ese sentido, el MTSK, al servir de herramienta para comprender el conocimiento del profesor que sustenta sus acciones, nos parece adecuado para explicar parcialmente el ETM idóneo del profesor. Del mismo modo, el ETM personal del profesor (esto es, el espacio de trabajo matemático en el que el profesor es hacedor de matemáticas) se ve directamente 
afectado por su conocimiento especializado, en especial en lo referente al dominio matemático.

Finalmente, una posible aportación a la interpretación de relaciones entre ETM y conocimiento del profesor, viene dada por la consideración de un espacio de referencia intermedio entre el determinado por el saber sabio y el ETM idóneo, el ETM de referencia del profesor. El MTSK del profesor (en sus dos subdominios -conocimiento matemático y conocimiento didáctico del contenido) incide en su ETM de referencia y la transformación de éste en el idóneo.

En todos los casos, estamos considerando los ETM (de referencia, idóneo y personal) del profesor. Además, coherentemente con nuestra visión dinámica del conocimiento del profesor, estos ETM del profesor se muestran cambiantes en el tiempo, lo que hemos hecho explícito en la figura 2.

Aventuramos que el MTSK, al permitir estudiar el conocimiento del profesor relativo a su acción y a la especificidad del contenido matemático, puede contribuir a que comprendamos mejor los ETM (de referencia, idóneo y personal) de dicho profesor, así como las relaciones que se establecen entre ellos. Además, desde una perspectiva de desarrollo profesional, puede aportar explicaciones a cómo evolucionan dichos ETM.

En sentido inverso, el marco del ETM posibilita profundizar en las relaciones entre el conocimiento especializado del profesor de matemáticas y las acciones de enseñanza que éste sustenta, en un esquema más amplio donde se incorpora el saber sabio y los espacios personales de los alumnos, así como la diferenciación entre planos y génesis.

Finalmente, consideramos que, ambos modelos, ETM y MTSK, comparten elementos que los hacen complementarios para analizar la actividad educativa en el aula. Cada uno de ellos centra su atención en uno de los agentes protagonistas del proceso de enseñanza y aprendizaje (alumno y profesor, respectivamente) y conciben la actividad matemática del aula como una actividad genuina de construcción y generación de conocimiento, por lo que son, además, epistemológicamente compatibles. Sería interesante profundizar en el papel que los subdominios del MTSK idóneo del profesor (a los que se puede acceder) poseen en la articulación entre los planos cognitivos y epistemológicos de los alumnos e indagar en el potencial comprensivo que la articulación de ambos modelos posee para la comprensión del proceso de enseñanza y aprendizaje.

\section{Referencias}


AZCÁRATE, P. El conocimiento profesional: naturaleza, fuentes, organización y desarrollo. Quadrante, Lisboa, v. 8, n. 1 y 2, p. 111-138, 1999.

BALL, D. L.; THAMES, M. H.; PHELPS, G. Content knowledge for teaching: what makes it special? Journal of Teacher Education, New York, v. 59, n.5, p. 389-407, nov/dic. 2008.

CARRILLO, J. et al.Determining specialised knowledge for mathematics teaching. In: UBUZ, B.; HASER, C.; MARIOTTI, M. A. (Ed.). Proceedings of the CERME 8. Middle East Technical Universitiy: Ankara, Turquía, 2013. p. 2985-2994.

ERNEST, P. The philosophy of mathematics education. London: The Falmer Press, 1991.

FERNÁNDEZ, S. et al.. Re-defining HCK to approach transition. In: PYTLAK, M.; ROWLAND, T; SWOBODA, E. (Ed.). Proceedings of the CERME 7. Rzeszów, Poland University of Rzeszów: ERME, 2011. p. 2640-2649.

GÓMEZ-CHACÓN, I. ; ROMERO, I. ; CARRILLO, J. Genèse et développement du travail mathématique : rôle de l'enseignant, du formateur et des interactions. In: FOURTH SYMPOSIUM ESPACE DE TRAVAIL MATHEMATIQUE, 4th, 2015, Madrid. Publications de l'ETM4. Madrid: Publicaciones del Instituto de Matemática Interdisciplinar, Universidad Complutense de Madrid, 2015, p. $405-410$.

KUZNIAK, A. L'espace de travail mathématique et ses genèses. Annales de didactique et des sciences cognitives, Strasbourg, v. 16, p. 9-24, 2011.

KUZNIAK, A.; RICHARD, P. Espacios de trabajo matemático. Puntos de vista y perspectivas. Revista Latinoamericana de Investigación en Matemática Educativa, México, v. 17, n. 4-I, p. 5-15, dic. 2014.

LEATHAM, K.R. Viewing mathematics teachers' beliefs as sensible systems. Journal of Mathematics Teacher Education, Las Vegas, v. 9, n. 1, p. 91-102, feb. 2006.

LIÑÁN, M.M.; MONTES, M.A.; CONTRERAS, L. C. Conocimiento sobre la recta de una maestra de tercer ciclo de educación primaria. In: SIMPOSIO DE LA SOCIEDAD ESPAÑOLA DE INVESTIGACIÓN EN EDUCACIÓN MATEMÁTICA, 19, 2015, Alicante. Investigación en Educación Matemática. Alicante: Universidad de Alicante, 2015. p.335-342.

LLINARES, S. El sentido numérico y la representación de los números naturales. In: CASTRO, E. (Ed.). Didáctica de la matemática en la educación primaria. Madrid: Síntesis, 2001. p. 151-176.

MARTÍNEZ, M. et al.. El conocimiento del horizonte matemático: más allá de conectar el presente con el pasado y el futuro. In: SIMPOSIO DE LA SOCIEDAD ESPAÑOLA DE INVESTIGACIÓN EN EDUCACIÓN MATEMÁTICA, 15, 2011, Ciudad Real. Investigación en Educación Matemática. Ciudad Real: Universidad de Castilla-La Mancha, 2011. p. 429-438.

MONTES, M. A. et al. From common and horizon knowledge to knowledge of topics and structures. In: CONGRESS OF EUROPEAN RESEARCH IN MATHEMATICS EDUCATION (CERME), 8 , 2013, Ankara. Proceedings of CERME 8. Ankara: Middle East Technical University, 2013, p. 29852994.

MONTOYA-DELGADILLO, E.; MENA-LORCA, A.; MENA-LORCA, J. Circulaciones y génesis en el espacio de trabajo matemático. Revista Latinoamericana de Investigación en Matemática

Educativa, México, v. 17, n. 4-I, p. 181-197, dic. 2014. 
NATIONAL COUNCIL OF TEACHERS OF MATHEMATICS (NCTM). Principios y estándares para la educación matemática. Traducción de la versión del 2000 del NCTM. Granada: S.A.E.M. Thales, 2004.

PONTE, J. P.; CHAPMAN, O. Mathematics teachers' knowledge and practice. In: GUTIERREZ, A.; BOERO, P. (Ed.). Handbook of research of the psychology of mathematics education: past, present and future. Rotterdam: Sense Publishing, 2006. p. 461-494.

RAUSCHER, J-C.; ADJIAGE, R. Espaces de travail et resolution d'un problème de modélisation. Revista Latinoamericana de Investigación en Matemática Educativa, México, v. 17, n. 4-I, p. 4164, dic. 2014.

SFARD, A. On the dual nature of mathematical conceptions: reflections on processes and objects as different sides of the same coin. Educational Studies in Mathematics, Dordrecht, v. 22, n. 1, p. 1-36, dic. 1991.

SHULMAN, L. Those who understand: knowledge growth in teaching. Educational Researcher, Washington, v. 15, n. 2, p. 4 - 14, feb. 1986.

SHULMAN, L. Knowledge and Teaching: Foundations of the New Reform. Harvard Educational Review, Cambridge, v. 57, n.1, p. 1-22, 1987.

THOM, R. Modern Mathematics: does it exist? In: HOWSON, A. G. (Ed.). Developments in mathematical education. Cambridge: Cambridge University Press, 1973. p.194-209.

THOMSON, A. Teachers' beliefs and conceptions: a synthesis of research. In: GROUWS, D.A. (Ed.). Handbook of research on mathematics teaching and learning. New York: McMillan \& NCTM, 1992. p. 127-146.

Submetido em Julho de 2015. Aprovado em Setembro de 2015. 